\title{
Nanochannels for diagnostic of thrombin-related diseases in human blood
}

\author{
Alfredo de la Escosura-Muñiz ${ }^{1}$, Wilanee Chunglok ${ }^{2}$, Werasak Surareungchai ${ }^{2}$, Arben \\ Merkoçi $i^{1,3 *}$ \\ ${ }^{1}$ Nanobioelectronics \& Biosensors Group, Institut Català de Nanotecnologia, Campus \\ UAB, Bellaterra (Barcelona), Spain. \\ ${ }^{2}$ School of Bioresources \& Technology, King Mongkut's University of Technology, \\ Thonburi, Bangkhuntien Campus, Bangkok, Thailand. \\ ${ }^{3}$ ICREA, Barcelona, Spain
}

Phone number: +34935868014 ; Fax number: +34935868020

E-mail: arben.merkoci@icn.cat 


\section{ABSTRACT}

A high sensitive voltammetric method for rapid determination of thrombin spiked in whole blood by taking advantage of both aptamer-based recognition and the use of a nanoporous membrane has been developed. The nanoporous membrane not only acts as platform for the thrombin recognition but also as filter of the micrometric components such as white and red blood cells, consequently minimizing matrix effects. The protocol involves a sandwich format in the inner walls (200 $\mathrm{nm}$ diameter) of an anodized alumina oxide filter membrane (AAO). The analytical signal, by DPV oxidation of $\left[\mathrm{Fe}(\mathrm{CN})_{6}\right]^{4-}$, is based on the blockage in the pores which affects the diffusion of $\left[\mathrm{Fe}(\mathrm{CN})_{6}\right]^{4-}$ to the screen-printed carbon electrotransducer (SPCEs) modified with the membrane. By labeling the anti-thrombin IgG with AuNPs followed by silver enhancement a greater passive signal enhancement in comparison to the membrane blockage has been observed. The contribution of both electrostatic/steric effects in this blockage due to the subsequent formation of the aptamerthrombin complex and the final sandwich assay is investigated. The efficiency of the system is also monitored by microscopic techniques. The resulted biosensing system allows

detecting thrombin spiked in whole blood at very low levels (L.O.D. $1.8 \mathrm{ng} \mathrm{mL}{ }^{-1}$ ) which are within the range of clinical interest for the diagnostic of coagulation abnormalities as well as pulmonary metastasis.

KEYWORDS: Nanopores; Nanochannels; Aptamer; Thrombin; Screen-printed carbon electrodes; Gold nanoparticles. 


\section{INTRODUCTION}

The detection of thrombin in blood is of great importance in clinical analysis. The thrombin-fibrinogen interaction is part of the physiological process of blood clotting and thrombin has also hormone-like properties when it is involved in thrombosis and platelet activation [Wolberg, 2007]. The concentration of thrombin in blood can vary considerably: it is not present in blood under normal conditions, but can reach low-micromolar concentrations during coagulation processes [Bichler et al., 1996]. Independently of the hemostatic process, thrombin circulates at the high-picomolar level in the blood of patients suffering from diseases known to be associated with coagulation abnormalities. Furthermore, thrombin is considered as a tumor marker useful for the diagnosis of pulmonary metastasis [Nierodzik et al., 2006]. For these both scenarios the development of simple (in comparison to ELISA, Western-blot, etc.) biosensing devices able to rapidly detect thrombin in human blood at picomolar $\left(\mathrm{ng} \mathrm{mL}^{-1}\right)$ levels would be of great interest.

Colorimetric [Chen et al., 2010] and amperometric [Thuelermann et al., 2009] biosensing strategies that use antibodies as receptors for the detection of thrombin in blood have already been reported. Although the high sensitivity and low detection limits achieved using the mentioned approaches these still do not solve the problems related to real blood sample analysis also the robustness (in terms of the receptor used) while thinking for future in-filed / point of care applications.

Aptamers are promising bioanalytical tools for rapid and sensitive protein detections, including thrombin analysis, and for developing of protein arrays [Mukhopadhay, 2005]. These synthetic nucleic acid sequences act as antibodies in binding proteins owing to their 
relatively easy isolation and modification, holding a high affinity and high stability [Hansen et al., 2006]. It is well-known that the single-stranded DNA oligonucleotide 5'GGTTGGTGTGGTTGG-3' (thrombin aptamer) acts as thrombin inhibitor. The mechanism of interaction consists in the binding of the aptamer to the anion-binding exosite, inhibiting thrombin's function by competing with exosite binding substrates fibrinogen and the platelet thrombin receptor [Paborsky et al., 1993].

This highly specific aptamer/thrombin binding interaction has been extensively approached to develop different biosensors for thrombin, taking advantage of the high sensitivity, selectivity, simple instrumentation, portability and cost effectiveness of the biosensing devices using different transduction techniques such as optical [Pavlov, et al., 2005], voltammetric [Suprun et al., 2008] and impedimetric [Zhang et al., 2009].

The combination of the aptamer-based electrochemical biosensors with the tools provided by the nanotechnology, such as the use of nanoparticle tags [Hianik et al., 2005; Heyduk et al., 2005] and multi-walled carbon nanotubes [Kara et al., 2010] with final detection by i.e. electrochemical impedance spectroscopy, has given rise to very sensitive biosensing systems for thrombin at even below the picomolar levels. However, in most cases a blood sample pre-treatment is required in order to remove red and blood cells as well as other components which can interfere in the specific thrombin detection.

In this context, nanoporous materials starting from Coulter counter [Coulter, 1953] and its evolution from micro- to nano- sized [Bezrukov et al., 1994] sensing pores present outstanding new features for biosensor development [Howorka et al., 2009; Hou et al., 2009; Martin et al., 2007]. The pioneered nanopore sensor consisted in a $\alpha$-hemolysin protein single channel embedded within a lipid bilayer membrane; an ionic current was 
passed through the channel, and analyte species bound inside the channels were detected as transient blocks in this current associated with translocation of the analyte through the channel-stochastic sensing [Bayley et al., 2001]. The drawback related to the fragility of the supported bilayer membrane that houses the nanopore was then solved building artificial nanopores embedded in a mechanically and chemically robust synthetic membranes [Gyurcsányi, 2008], being the anodized alumina oxide (AAO) one of the most commonly used for these purposes.

In addition to their extensive study for DNA sequencing, the blockage of the ion current in AAO nanochannel arrays by a bioreaction has also been approached by our group and others for ssDNA [Vlassiouk et al., 2005; De la Escosura Muñiz et al., 2010a] and protein [De la Escosura-Muñiz et al., 2010b] detection, even in blood samples [De la EscosuraMuñiz et al., 2011], taking advantage of their properties not only as sensing platforms but also as filters of micrometric components of the blood.

Here we present a novel aptamer-based biosensor for thrombin detection using AAO membranes, containing pores of $200 \mathrm{~nm}$ in diameter, and screen-printed carbon electrotransducers (SPCEs). The dual electrostatic/steric blockage in the pores due to the specific binding of thrombin and the further amplification in a sandwich assay using gold nanoparticle tags using the system $\left[\mathrm{Fe}(\mathrm{CN})_{6}\right]^{4-/ 3-}$ as red-ox indicator is investigated. The nanoparticle-based amplification strategy in combination with the filtering properties of the membranes allows to specifically detect thrombin in whole blood at picomolar levels, which is within the range of clinical interest. 


\section{EXPERIMENTAL SECTION}

\subsection{Apparatus and electrodes}

Anodized alumina oxide filter membranes (Whatman anodisc AAO filters, $13 \mathrm{~mm}$ diameter; $60 \mu \mathrm{m}$ thick containing 200 and $20 \mathrm{~nm}$ pores of $1 \times 10^{9} \mathrm{~cm}^{-2}$ density) were purchased from Scharlab (Spain). The electrochemical transducers used were homemade screen-printed carbon electrodes (SPCEs) and the measurements were performed using a home-made methacrylate cell connected to an Autolab 20 (Eco-chemie, The Netherlands). See the detailed SPCE fabrication procedure, pictures of the obtained sensors and of the electrochemical cell set-up as well as details of the optical apparatus used at the Supplementary material.

\subsection{Reagents and solutions}

A 5' amino modified DNA aptamer, selective to human alpha thrombin: ${ }^{\prime} \mathrm{NH}_{2^{-}}$ GGTTGGTGTGGTTGG-3' was purchased from Alpha DNA (Canada). Oligonucleotide solution was prepared in TE buffer, $\mathrm{pH} 8(10 \mathrm{mM}$ Tris- $\mathrm{HCl}$ buffer solution, $1 \mathrm{mM}$ in EDTA) and maintained at $-20^{\circ} \mathrm{C}$. Working solutions were made in $0.1 \mathrm{M}$ Tris, $\mathrm{pH} 7.2$ buffer and conserved at $4{ }^{\circ} \mathrm{C}$.

Thrombin from human plasma (T6884), tyrosinase (T3824) and goat anti-rabbit IgG-FITC (F0382) were purchased from Sigma (Spain). Human alpha thrombin and tyrosinase working solutions were prepared in $0.01 \mathrm{M}$ phosphate buffered saline, $\mathrm{pH} 7.4$ (PBS). Rabbit anti-human thrombin was purchased from Abcam (Spain).

All the chemical reagents were purchased from Panreac, Sigma and Fluka (see Supplementary Material). 
Lyophilised human whole blood (certified reference material, Community Bureau of Reference -BCR ${ }^{\circledR}$ 635-, preserved in sodium-EDTA anticoagulant) was purchased from Sigma (Spain) and reconstituted in Milli-Q water, following the manufacturer indications. Human Alpha Thrombin was spiked and homogenized in the resulting blood sample.

Suspensions of AuNPs (80 nm sized) were purchased from BBInternational (UK). The conjugation of AuNPs to anti-human thrombin antibodies was performed according to a previously optimized procedure (see the Supplementary material). Zeta potential of the AuNPs before and after their conjugation with antibodies was determined with a Malvern Zetasizer Nano-ZS (Malvern Instruments Ltd., UK) according to the manufacturer's recommendations.

Silver enhancement was performed by using an LI Silver Enhancement Kit (Nanoprobes Inc., USA). The two components of the LI Silver Enhancement Kit were stored at $4{ }^{\circ} \mathrm{C}$ and a reactive mixture was prepared at $24^{\circ} \mathrm{C}$ just before use. The stability-no selfnucleation - is guaranteed at this temperature over $45 \mathrm{~min}$.

\subsection{Methods}

\section{Zeta Potential Measurements}

A $1 \mu \mathrm{L}$ suspension of AuNPs (the same as for the anti-thrombin/AuNPs conjugates) was diluted in $1 \mathrm{~mL}$ of PBS buffer, vortexed, and transferred into a $4 \mathrm{~mL}$ polystyrene cuvette (FB55143, Fisher Scientific). The data were collected and analyzed with the Dispersion Technology software 4.20 (Malvern) producing diagrams for the zeta potential as a distribution versus total counts. 
AAO filter membranes functionalization and immobilization of aptamers inside the nanochannels

The aptamer immobilization in the inner walls of the nanochannels was performed following an experimental procedure previously optimized for oligonucleotide sequences [De la Escosura-Muñiz et al., 2010a], consisting first in the silanization and generation of carbonyl groups in the walls of the nanochannels, Then the aptamer was bound through the peptide bond by placing $30 \mu \mathrm{L}$ of $1 \mathrm{mg} \mathrm{mL}^{-1}$ solution of the amino-modified aptamer on the filtering side of the membranes and keeping in a moist atmosphere overnight at $4^{\circ} \mathrm{C}$ in order to avoid their dryness. To achieve such conditions, the membranes were placed in a glass slide inside a closed plastic box filled with water. The same experimental set-up was used for all the biological incubations.

See the detailed membranes silanization procedure as well as a scheme of the full process at the Supplementary material.

\section{Aptamer/thrombin interaction inside the nanochannels}

After thorough washing in PBS buffer, $30 \mu \mathrm{L}$ of thrombin solution was placed on the filtering side of the membrane and left there for $60 \mathrm{~min}$ at room temperature (see the optimization of the reaction time in the Supplementary material).

The optimizations of the analytical method were performed using thrombin solutions in PBS and then the optimized method was applied for thrombin spiked in human blood.

Blank assays were performed following the same protocol but using tyrosinase in PBS buffer instead of thrombin. In the case of the human blood samples analysis, the blank assays corresponded to the whole blood without added thrombin. 


\section{Sandwich assay and silver amplification}

After washing in PBS buffer, the sandwich assay was completed by placing $30 \mu \mathrm{L}$ of the AuNP labeled antibody (rabbit anti-thrombin/AuNPs) on the filtering side of the membranes, and left there for $60 \mathrm{~min}$ at room temperature. After that, the membranes were thoroughly washed in deionized water (to avoid the further silver precipitation with chloride or phosphate of other anions that can be present in the sample or the buffers) and then $30 \mu \mathrm{L}$ of the LI Silver Enhancement reactive mixture were placed on their filtering side and left there for $20 \mathrm{~min}$ at $24{ }^{\circ} \mathrm{C}$, in order to selective deposit silver around the AuNPs tags. Finally the membranes were washed again with Milli-Q water.

\section{Nanoporous cell set-up and electrochemical detection}

AAO filter membranes were fixed onto the screen-printed carbon electrodes (SPCEs) by a physical attachment inside a methacrylate cell, as detailed at the Supplementary Material.

The $200 \mu \mathrm{L}$ electrolytic cell defined was filled with $100 \mu \mathrm{L}$ of $1 \mathrm{mM} \mathrm{K}_{3}\left[\mathrm{Fe}(\mathrm{CN})_{6}\right]$ in $0.1 \mathrm{M}$ $\mathrm{NaNO}_{3}$ solution and a pre-treatment process at $-0.55 \mathrm{~V}$ during $30 \mathrm{~s}$ was carried out in order to have all the $\mathrm{Fe}$ ions in the form of $\mathrm{Fe}$ (II). Immediately after, differential pulse voltammetry (DPV) by scanning from $-0.3 \mathrm{~V}$ to $+0.3 \mathrm{~V}$ (resulting in an analytical signal

due to oxidation of $\left[\mathrm{Fe}(\mathrm{CN})_{6}\right]^{4-}$ to $\left[\mathrm{Fe}(\mathrm{CN})_{6}\right]^{3-}$ at approximately $\left.+0.1 \mathrm{~V}\right)$ was performed. A step potential of $10 \mathrm{mV}$, modulation amplitude of $50 \mathrm{mV}$ and a scan rate of $33.5 \mathrm{mV} \mathrm{s}^{-1}$ were applied during the measurements in a non-stirred solution. The measurements were made in ambient room air and temperature.

Each measurement was performed with a new membrane and a new SPCE. 


\section{RESULTS AND DISCUSSION}

\subsection{Label-free specific detection of thrombin: steric blockage and charge repulsion effects}

The label-free selective detection of thrombin using AAO nanoporous membranes is achieved, ascribed to the partial blockage in the diffusion of electroactive species $\left(\left[\mathrm{Fe}(\mathrm{CN})_{6}\right]^{4-}\right.$ ions corresponding to the ferrocyanide used as red-ox indicator) through the nanochannels to the screen-printed carbon electrotransducer (SPCE) due to the formation of the complex aptamer/thrombin. This blockage gives rise to a decrease in the voltammetric oxidation signal of $\left[\mathrm{Fe}(\mathrm{CN})_{6}\right]^{4-}$ to $\left[\mathrm{Fe}(\mathrm{CN})_{6}\right]^{3-}$ chosen as analytical signal, allowing the detection and quantification of thrombin as schematized in Scheme 1.

\section{Preferred position for Scheme 1}

The reasons of the decrease in the voltammetric signal observed after the formation of the aptamer/thrombin complex can be attributed to a dual effect based on both steric blockage and charge repulsion effects. The aptamer by itself produces a certain decrease in the signal (Figure 1A, voltammogram b) compared with signal from the bare membranes (Figure 1A, voltammogran a), being this the background signal for the sensing experiments.

\section{Preferred position for Figure 1}

The decrease in the voltammetric signal can be explained mainly considering charge repulsion effects. As the oligonucleotide sequence of the aptamer is negatively charged, this produces a repulsion to the negative charge of the $\left[\mathrm{Fe}(\mathrm{CN})_{6}\right]^{4-}$ ions contained in the red-ox indicator, being thus the ions diffusion to the electrode reduced and the later voltammetric re-oxidation consequently decreased. In this case, the small size of the aptamer (considering 
the size of a 15-mer ssDNA: approximate diameter of $1.84 \mathrm{~nm}$ and length of $0.27 \mathrm{~nm}$ ) indicates that the steric blockage in the diffusion of the $\left[\mathrm{Fe}(\mathrm{CN})_{6}\right]^{4-}$ ions to the electrode is probably minimum being the charge repulsion between the redox indicator and the aptamer the most important factor.

When the sample containing thrombin is added to the nanoporous membranes, the formation of the complex aptamer/thrombin increases both effects, allowing the thrombin recognition and quantification. At $\mathrm{pH}$ of the redox indicator medium $\left(1 \mathrm{mM} \mathrm{K}_{3}\left[\mathrm{Fe}(\mathrm{CN})_{6}\right] /\right.$ $0.1 \mathrm{M} \mathrm{NaNO}$, $\mathrm{pH}:$ 7.5) the thrombin is also negatively charged (the isoelectric point is around 7 [Schon et al., 2005]) and this charge enhances the electrostatic repulsion to the $\left[\mathrm{Fe}(\mathrm{CN})_{6}\right]^{4-}$ ions, causing lower voltammetric signals (Figure 1A, voltammogram c). In addition, since the size of thrombin is around $4 \mathrm{~nm}$ [Rinker at al., 2008; Thiel, 2004], its presence inside the nanochannels also exerts a steric blockage considerably higher than that exerted by the free aptamer. This important steric contribution was evidenced by the better sensitivity obtained for the membranes with smaller pore sizes $(20 \mathrm{~nm})$ in comparison with the obtained for the membranes containing pores of $200 \mathrm{~nm}$ in diameter (LOD of around $100 \mathrm{ng} \mathrm{mL} L^{-1}$ and $10 \mu \mathrm{g} \mathrm{mL}^{-1}$ respectively) as shown in Figure 1B. However, in spite of the better sensitivity obtained, the membranes with $20 \mathrm{~nm}$ sized pores were discarded since the very small pore size impelled unspecific blockages even by the salt crystals of the red-ox indicator medium.

The specificity of the biosensing system was evaluated using tyrosinase as negative control, no significant changes in the analytical signal observed, evidencing that this protein is not neither recognized by the aptamer nor adsorbed on the inner walls of the nanochannels in a non-specific way (see the Supplementary material). Tyrosinase is usually used as control 
for aptamer-based thrombin recognition assays, due to their similar size. Furthermore, tyrosinase has been shown to inactivate thrombin and so to modify fibrinogen [Sizer, 1952]. In addition its presence in peripheral blood at abnormal levels has been related with the malignant melanoma prognosis [Carrillo et al., 2006].

\subsection{Signal amplification using AuNPs tags and silver enhancement}

In order to improve the detection limits, an amplification strategy based on the use of antibodies labeled with AuNPs to complete a sandwich assay after the thrombin capturing by the aptamer was followed. The availability of the thrombin molecules to be recognized by rabbit anti-thrombin antibodies after their capture by the aptamer inside the nanochannels was first checked by confocal microscopy, using specific secondary antirabbit antibodies labeled with a fluorescence marker (FITC). As it can be seen in Figure 2A, fluorescence is observed not only on the surface but also inside the nanochannels, demonstrating the availability of the epitopes of the thrombin to be recognized by such antibodies.

\section{Preferred position for Figure 2}

The amplification of the analytical signal (decrease in the voltammetric peak current) was then accomplished performing the sandwich assay with AuNPs (80 nm sized) labels (figure

1A, voltammogram d), allowing to improve the LOD of thrombin from $10 \mu \mathrm{g} \mathrm{mL} \mathrm{L}^{-1}$ to around $10 \mathrm{ng} \mathrm{mL}^{-1}$ (figure $1 \mathrm{~B}$ ).

The explanation of such LOD improvements of around three orders of magnitude more sensitive than for the label-free assay can be found again in the combination of electrostatic/steric blockage in the diffusion of the $\left[\mathrm{Fe}(\mathrm{CN})_{6}\right]^{4-}$ ions through the nanopores to reach the electrode. The large size of the AuNPs $(80 \mathrm{~nm})$ increases in a high extent the 
physical blockage of the diffusion of the electroactive ions to the electrode. In order to elucidate the electrostatic contribution to this blockage it was taken advantage of the zeta potential technique. Zeta potential technique has been recently reported as an efficient tool which allows the monitoring and analysis of chemical modifications on the surface of nanoparticles, with minimal sample preparation [Thielbeer et al., 2011]. The zeta potential is generated when charged particles are dispersed in a medium and gives rise to the formation of an electric potential between the surface of the particle and the medium, because the surface charge of the particle attracts counterions. A double layer is formed at the interface of the particle; the first layer (so called Stern-layer) contains ions directly adsorbed to the surface, and this layer is surrounded by a more diffuse second layer of mobile ions attracted by the surface charge. The potential between the diffuse layer and the medium is called the zeta potential. The easily measurable zeta potential (in contrast with the extreme difficulty to measure the potential of the Stern-Layer) has consequently been used to obtain information concerning the particle surface charge, chemical modifications and also stability of colloid suspensions [Butt et al., 2003]. A high zeta potential (positive or negative) confers stability since the solution or dispersion resists aggregation. When the absolute value of the zeta potential is low, attraction exceeds repulsion and the dispersion breaks and flocculates. Due to all that, it has been defined that nanoparticle suspensions begin to be moderated stable for absolute zeta potential values higher than $10 \mathrm{mV}$. So both unmodified AuNPs and anti-thrombin/AuNPs were analyzed by zeta potential, following the experimental procedure detailed in materials and methods section. As can be observed in the graph of figure $2 \mathrm{~B}$, the value of the zeta potential of the AuNP suspensions was around $-30 \mathrm{mV}$, indicating a very negative surface charge and a good stability. This negative charge is probably due to the citrate ions (used as reductive agents in the AuNPs 
synthesis) which are recovering and stabilizing the AuNPs. After the conjugation with the antibody, this value shifted to $-17 \mathrm{mV}$ evidencing the probable substitution of the citrate ions on the surface of the AuNPs by the antibodies, giving rise to negative charged conjugates which are still within the stability range of values.

Consequently, when the sandwich assay is performed with the anti-thrombin/AuNPs conjugate, the negative charge of the conjugate is added to that of the thrombin, increasing the electrostatic repulsion of the $\left[\mathrm{Fe}(\mathrm{CN})_{6}\right]^{4-}$ ions as schematized in the cartoon of Figure 2B.

Finally, the catalytic activity of the AuNPs on the silver reduction was also approached to selectively deposit silver around the AuNPs, forming a shell that grows up reaching approximately the same size of the nanochannel's diameter $(200 \mathrm{~nm})$. As indicated by Figure 3 (right), silver enhanced AuNPs perfectly fit inside the channels allowing a highly enhanced steric blockage to the diffusion of the $\left[\mathrm{Fe}(\mathrm{CN})_{6}\right]^{4-}$ ions.

\section{Preferred position for Figure 3}

The voltammetric peak currents in the presence of silver enhanced AuNPs are the lowest registered (Figure 1A, voltammogram e) evidencing the predominance of the highly enhanced steric blockage in this case. It is also noticed the presence of a peak corresponding to the re-oxidation of the deposited $\mathrm{Ag}^{0}$ to $\mathrm{Ag}^{+}$at around $0.0 \mathrm{~V}$ which does not affect the peak at approximately $+0.1 \mathrm{~V}$ and that is not considered for analytical

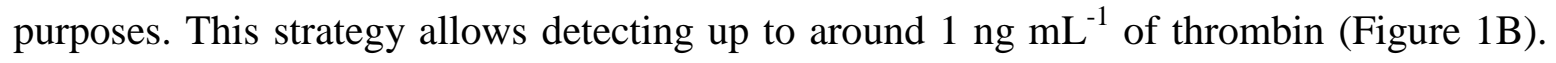
The value meets the range of clinical interest. 


\subsection{Thrombin analysis in human blood}

As it has been mentioned, the detection of thrombin in whole human blood is of great importance in clinical analysis, not only for the diagnosis of coagulation problems also for its consideration as a tumor marker. As it has been recently reported by our group, one of the main advantages of the use of nanoporous membranes as biosensing platforms consists in their ability to act also as filter of biomolecules with sizes larger than the pore diameter [De la Escosura-Muñiz et al., 2011]. This filter-like effect minimizes matrix effects upon the analytical signal and allows the direct detection of proteins in blood, without any pretreatment of the sample (centrifugation/re-suspension processes, etc.).

Considering that, human blood solutions were spiked with thrombin (see materials and methods section) and the same experimental procedure as the one followed for the solutions prepared in buffer was carried out. The predicted filtering effect of the nanoporous membranes was first evidenced by SEM analysis. As it can be clearly seen in Figure 4, the white and red blood cells do not enter into the membranes because of the size-exclusion effect, while most of the pores remain free.

\section{Preferred position for Figure 4}

Thrombin can penetrate through these pores, being captured inside the nanochannels by the aptamer. It is important to highlight that the optimized aptamer/thrombin reaction time (60 min) is low enough to guarantee that, thanks to the chelate effect of the EDTA anticoagulant, the $\mathrm{Ca}^{2+}$ ions in blood remain complexed and are not able to exert their effect in the prothrombin conversion to thrombin [Mitchell, 2009]. Reaction times longer than 2 hours would give rise to the starting of the coagulation process (even in the presence of the anticoagulant) and the presence of no thrombin added (note also that due to the special 
conditions of the sample preparation for the SEM analysis, the observed sample characteristics are not the same as for the electrochemical measurements).

The thorough washing of the membrane (after incubations with $30 \mu \mathrm{L}$ of whole blood with spiked thrombin) allows removing of the cells and continuing of the later incubation with AuNPs labeled antibodies and the silver enhancement without matrix interferences. The selective formation of the silver shells inside the nanochannels was clearly observed too by SEM (Figure 3, right). When the assay is performed for blood without added thrombin, silver crystals are not observed (Figure 3, left) demonstrating the specificity of the assay as well as of this amplification strategy.

Furthermore, it must be highlighted that the quantity of silver shells observed along the nanochannels is much higher than the observed, i.e. in previous works for sandwich immunoassays using both capturing and signaling antibodies [De la Escosura-Muñiz et al., 2011]. This can be due to the fact that in the case of the sandwich immunoassays, after the recognition by the capturing antibody the number of the epitopes available in the antigen for the later recognition by the signaling antibody is limited; a competition between both antibodies (capturing and signaling antibodies) for the epitopes (being this more critical for small proteins) occurs. However, in the case of the aptamer based assays, the aptamerprotein interaction doesn't affect the protein epitopes, keeping these more available for the later interaction with the signaling antibody [He et al., 2007; Kang et al., 2008]. This gives rise to the presence of a higher quantity of labeled antibodies for each thrombin molecule as observed through the high abundance of silver crystals in the inner walls of the nanochannels. 
These optical observations are in correlation with the electrochemical results. As it can be observed in Figure 5A, the voltammetric peak currents registered at approximately $+0.1 \mathrm{~V}$ corresponding to the oxidation of $\left[\mathrm{Fe}(\mathrm{CN})_{6}\right]^{4-}$ to $\left[\mathrm{Fe}(\mathrm{CN})_{6}\right]^{3-}$ decrease when the thrombin concentration in blood increases.

\section{Preferred position for Figure 5}

It is also noticed that the peak corresponding to the re-oxidation of the deposited $\mathrm{Ag}^{0}$ to $\mathrm{Ag}^{+}$at around $0.0 \mathrm{~V}$ is always present but does not affect the analytical peak at $+0.1 \mathrm{~V}$. The presence of this silver-related peak and its current intensity corroborates the explanations given for the sensing principle using enhancement method. For the highest concentration of thrombin in blood, the $\mathrm{Ag}^{0}$ re-oxidation peak reaches a maximum value due to the formation of a higher number of silver crystals inside the channels. In contrast, for the lowest concentration of thrombin, the small quantity of silver crystals gives rise to the lowest peak current intensity. However, the irreproducibility observed for the intermediate thrombin concentrations (probably due to the different distances between the silver crystals and the electrode surface for the different membranes and electrodes) does not allow to consider this peak for analytical purposes. When the assay is performed for a blood sample without thrombin, the silver peak is not observed (Figure 5A, dashed line) and the value of the peak current at $+0.1 \mathrm{~V}$ is only a little bit lower than the background signal defined in previous sections (signal corresponding to obtained for the membranes with only aptamer), indicating a minimum unspecific absorption of some of the blood proteins (albumins, globulins and fibrinogen, all of them presented at grams per liter levels) in the inner walls of the membranes. 
As it can be observed in Figure 5B, there is a linear relationship between the analytical voltammetric peak current at $+0.1 \mathrm{~V}$ and the thrombin concentration in the range of 2-100 $\mathrm{ng} \mathrm{mL} \mathrm{m}^{-1}$, with a correlation coefficient of 0.997 , adjusted to the following equation:

$$
\text { peak current }(\mu \mathrm{A})=-0.003\left[\text { thrombin }\left(\mathrm{ng} \mathrm{mL}^{-1}\right)\right]+0.4(\mathrm{n}=3)
$$

The reproducibility responses of $60 \mathrm{ng} \mathrm{mL}^{-1}$ thrombin, $\mathrm{n}=10$, give a relative standard deviation (RSD) of $8 \%$ and the limit of detection calculated as the concentration of thrombin corresponding to three times the standard deviation of the estimate was $1.8 \mathrm{ng}$ $\mathrm{mL}^{-1}$. This value, as well as the linear response range, are comparable and even better than those obtained with other aptamer-based optical [Chen et al., 2010] and electrochemical [Li et al., 2010] biosensors for the detection of thrombin in whole blood within the above mentioned range of clinical interest, opening the way to future applications in diagnostics.

\section{CONCLUSIONS}

A simple aptamer modified nanochannel for thrombin detection in whole blood is presented. It is based on electrochemical detection using DPV of $\left[\mathrm{Fe}(\mathrm{CN})_{6}\right]^{4-} /\left[\mathrm{Fe}(\mathrm{CN})_{6}\right]^{3-}$ system used as indicator. The detection principle and the effect of the electrostatic interactions between thrombin and the aptamer modified inner walls of the nanochannel including the steric effects (thrombin aptamer, thrombin, nanoparticle and nanochannel sizes) are also clarified.

The developed nanochannels/aptamer based sensing system is applied for the direct detection of thrombin spiked in a whole blood sample. The efficiency of the nanoporous platform to act as a filtering layer avoiding the possible interferences of the sample matrix such as the red and white blood cells beside other micro components is checked through 
SEM observations beside electrochemical signals. The efficiency of the thrombin aptamer to selectively detect thrombin even in the presence of the high concentrations of blood proteins (albumins, globulins and fibrinogen, all them present at grams per liter levels) was also demonstrated. Furthermore, it is previewed an improvement in the performance of the biosensing system by tunning the thickness of the membranes as well as the pores density and diameter, bringing advantages such as lower protein detection limits.

The developed platform is previewed to bring advantages in several other proteins detections from complex matrix/samples. The high stability / robustness, selectivity and sensitivity of aptamers combined with the simplicity of nanochannels based platform may bring novel interesting applications for several other diagnostics, safety and security applications.

\section{ACKNOWLEDGMENTS}

We acknowledge MICINN (Madrid) for the projects PIB2010JP-00278 and IT2009-0092, the E.U.'s support under FP7 contract number 246513 "NADINE”' and the NATO Science for Peace and Security Programme's support under the project SfP 983807. W.C. acknowledges a Ph.D. scholarship from the Royal Golden Jubilee Project of the Thailand Research Fund. 


\section{REFERENCES}

Bayley, H., Cremer, P.S. 2001. Nature, 413, 226-230.

Bezrukov, S.M., Vodyanoy, I., Parsegian, V.A. 1994. Nature 370, 279-281.

Bichler, J., Heit, J.A., Owen, W.G. 1996. Thromb. Res. 84, 289-294.

Butt, H.J., Graf, K., Kappl, M. 2003. Physics and Chemistry of Interfaces. Wiley-VCH Verlag GmbH \& Co KGaA, Weinheim.

Carrillo, E., Prados, J., Marchal, J.A., Boulaiz, H., Martínez, A., Rodríguez-Serrano, F., Caba, O., Serrano, S., Aránega, A. 2006. Dis. Markers 22(3), 175-181.

Coulter, W.H. 1953. U.S. Patent, No. 2, 656, 508.

Chen, C.K., Huang, C.C., Chang, H.T. 2010. Biosens. Bioelectron. 25, 1922-1927.

De la Escosura-Muñiz, A., Mekoçi, A. 2010a. Chem. Commun. 46, 9007-9009.

De la Escosura-Muñiz, A, Merkoçi, A. 2010b. Electrochem. Comm. 12, 859-863.

De la Escosura-Muñiz, A., Merkoçi, A. 2011. Small 7, 675-682.

Gyurcsányi, R.E. 2008, Trends Anal. Chem. 27, 627-639.

Hansen, J.A., Wang, J., Kawde, A.N., Xiang, Y., Gothelf, K.V., Collins, G. 2006. J. Am. Chem. Soc. 128, 2228-2229.

He, P., Shen, L., Cao, Y., Li, D. 2007. Anal. Chem. 79, 8024-8029.

Heyduk, E., Heyduk, T. 2005. Anal. Chem. 77, 1147-1156.

Hianik, T., Ostatná, V., Zajacová, Z., Stoikova, E., Evtugyn, G. 2005. Bioorg. Med. Chem. Lett. 15, 291-295. 
Howorka, S., Siwy, Z. 2009. Chem. Soc. Rev. 38, 2360-2384.

Hou, X., Jiang, L. 2009. ACS Nano 3(11), 3339-3342.

Kang, Y., Feng, K.J., Chen, J.W., Jiang, J.H., Shen, G.L., Yu, R.Q. 2008. Bioelectrochem. $73,76-81$.

Kara, P., de la Escosura-Muñiz, A., Maltez-da Costa, M., Guix, M., Ozsoz, M., Merkoçi, A. 2010. Biosens. Bioelectron. 26, 1715-1718.

Li, L., Zhao, H., Chen, Z., Mu, X., Guo, L. 2010. Anal. Bioanal. Chem. 398, 563-570.

Martin, C.R., Siwy, Z.S. 2007. Science 317, 231-332.

Mitchell, R.N. 2009. Kumar: Robbins and Cotran Pathologic Basis of Disease. Professional Edition, 8th Ed. Saunders (Elsevier). Ch.4 Haemodynamic diseases.

Mukhopadhyay, R. 2005. Anal. Chem. 77, 114A-118A.

Nierodzik, M.L., Karpatkin, S. 2006. Cancer Cell 10, 355-362.

Paborsky, L., McCurdy, S., Griffin, L., Toole, J., Leung, L. 1993. J. Biol. Chem. 268, 20808-20811.

Pavlov, V., Shlyahovsky, B., Willner, I. 2005. J. Am. Chem. Soc. 127, 6522-6523.

Rinker, S., Ke, Y., Liu, Y., Chhabra, R., Yan, H. 2008. Nat Nanotechnol. 3(7), 418-422.

Schon, P., Degefa, T.H., Asaftei, S., Meyer, W., Walder, L. 2005. J. Am. Chem. Soc. 127, 11486-11496.

Sizer, I.W. 1952. Science 116, 275-276.

Suprun, E., Shumyantseva, V., Bulko, T., Rachmetova, S., Radko, S., Bodoev, N., Archakov, A. 2008. Biosens. Bioelectron. 24, 825-830. 
Thiel, K. 2004. Nature Biotech. 22(6), 649-651.

Thielbeer, F., Donaldson, K., Bradley, M. 2011. Bioconj. Chem. 22, 144-150.

Thuerlemann, C., Haeberli, A., Alberio, L. 2009. Clin. Chem. 55:3, 505-512.

Vlassiouk, I., Takmakov, P., Smirnov, S. 2005. Langmuir 21, 4776-4778.

Wolberg, A.S. 2007. Blood Rev. 21, 131-142.

Zhang, Z., Yang, W.,Wang, J., Yang, C., Yang, F., Yang, X., 2009. Talanta 78, 1240-1245.

\section{FIGURE CAPTIONS}

Scheme 1. Scheme of the sensing principle. The presence of thrombin in the sample (middle) difficult the diffusion of the $\left[\mathrm{Fe}(\mathrm{CN})_{6}\right]^{4-}$ ions through the nanoporous membranes to the electrode, due to both steric/electrostatic effects in comparison with the sample without thrombin (left), resulting in a decrease in the voltammetric signal of the $\left[\mathrm{Fe}(\mathrm{CN})_{6}\right]^{4-}$ $/\left[\mathrm{Fe}(\mathrm{CN})_{6}\right]^{3-}$ system. Both effects are enhanced doing a sandwich assay with AuNP labeled antibodies and the steric effect is highly increased depositing silver around the AuNPs (right).

Fig. 1. (A) DPVs registered in $1 \mathrm{mM} \mathrm{K}_{3}\left[\mathrm{Fe}(\mathrm{CN})_{6}\right] / 0.1 \mathrm{M} \mathrm{NaNO}$ for the $\mathrm{SPCE}$ covered with unmodified AAO filter membranes of $200 \mathrm{~nm}$ pore size (a) and for membranes modified with the aptamer before (b) and after (c) the reaction with $20 \mu \mathrm{g} \mathrm{mL}^{-1}$ of thrombin in PBS buffer (label-free assay); and after the reaction with $50 \mathrm{ng} \mathrm{mL}^{-1}$ of thrombin in PBS and sandwich assays performed with anti-thrombin/AuNPs (d) and after the silver enhancement (e). DPV parameters: pre-concentration potential: $-0.3 \mathrm{~V}$; pre-concentration time: $30 \mathrm{~s}$; step potential: $10 \mathrm{mV}$; modulation amplitude: $50 \mathrm{mV}$; scan rate: $33.5 \mathrm{mV} \mathrm{s}$. 
(B) Comparison of the limits of detection of thrombin obtained for the label-free assay for both membranes of $200 \mathrm{~nm}$ and $20 \mathrm{~nm}$ pore sizes and for the sandwich assays performed with AuNPs tags for the membranes of $200 \mathrm{~nm}$ pore sizes without and with silver enhancement.

Fig. 2 (A) Confocal microscopy images of an AAO filter membrane of $200 \mathrm{~nm}$ pore size after the aptamer modification, reaction with thrombin, sandwich assay with rabbit antithrombin antibodies and secondary reaction with anti-rabbit antibodies labeled with FITC. Plan view (a) and planes until $2 \mu \mathrm{m}$ in depth from the top surface $(\mathrm{b}, \mathrm{c})$ and from the bottom surface $\left(b^{\prime}, c^{\prime}\right)$. (B) (Up) Diagram for the zeta potential as a distribution versus total counts for a dispersion of AuNPs before and after the conjugation with anti-thrombin IgG and a dispersion of the conjugate anti-thrombin/AuNPs; (Down) Schematic of the enhancement in the electrostatic repulsion to the $\left[\mathrm{Fe}(\mathrm{CN})_{6}\right]^{4-}$ ions diffusion into the nanochannels due to the negatively charged AuNPs tags in a sandwich assay.

Fig. 3. SEM (cross-sectional view) images of AAO filter membranes of $200 \mathrm{~nm}$ pore size modified with the aptamer and left to react with a blood sample containing $100 \mathrm{ng} \mathrm{mL}^{-1}$ of spiked thrombin (Right) and a blood sample without spiked thrombin (Left). The sandwich assay is then completed with anti-thrombin/AuNPs followed by silver enhancement (white silver crystals are observed). Inset cartoons correspond to schemes of the involved processes.

Fig. 4. SEM (top view) images of different zones of an AAO filter membrane of $200 \mathrm{~nm}$ pore size on which a $30 \mu \mathrm{L}$ drop of blood was deposited and treated following the procedure detailed in the materials and sections method. White and red blood cells as well as the nanopores are observed. (Note that these images are representative of what happens, 
although the morphology of the cells is altered due to the sample treatment necessary for the SEM analysis).

Fig. 5. (A) DPVs registered $1 \mathrm{mM} \mathrm{K}_{3}\left[\mathrm{Fe}(\mathrm{CN})_{6}\right] / 0.1 \mathrm{M} \mathrm{NaNO}$ following the optimized experimental procedure for blood samples containing spiked thrombin at concentrations of (up to down): 0 (dashed line), 2, 20, 60, 80 and $100 \mathrm{ng} \mathrm{mL}^{-1}$. DPV parameters are the same as those in figure 1A. (B) Effect of the concentration of thrombin spiked in blood on the voltammetric peak current of oxidation of $\left[\mathrm{Fe}(\mathrm{CN})_{6}\right]^{4-}$ to $\left[\mathrm{Fe}(\mathrm{CN})_{6}\right]^{3-}$ (approx. $\left.+0.1 \mathrm{~V}\right)$ chosen as analytical signal. 


\section{SCHEME 1}

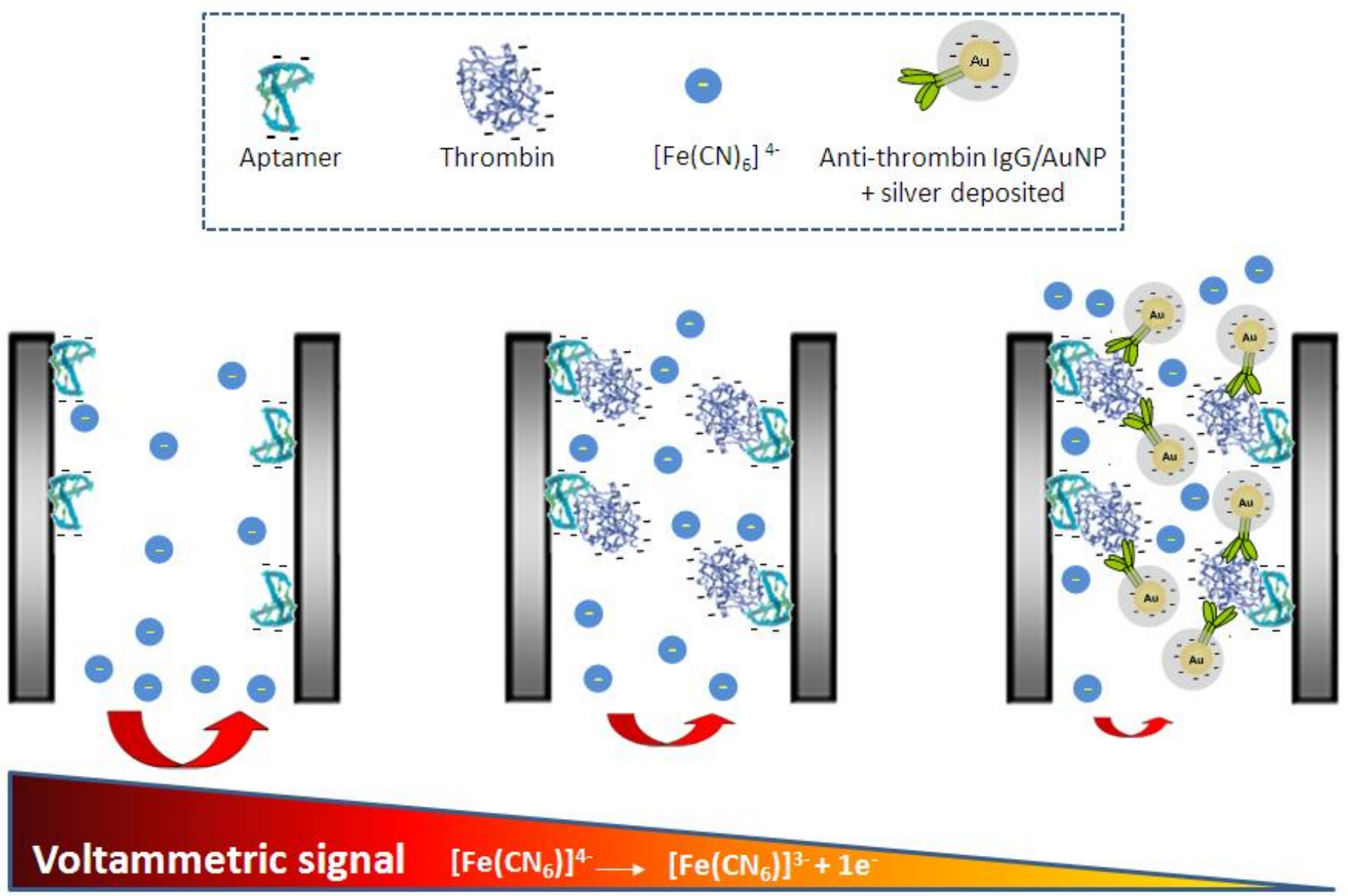




\section{FIGURE 1}
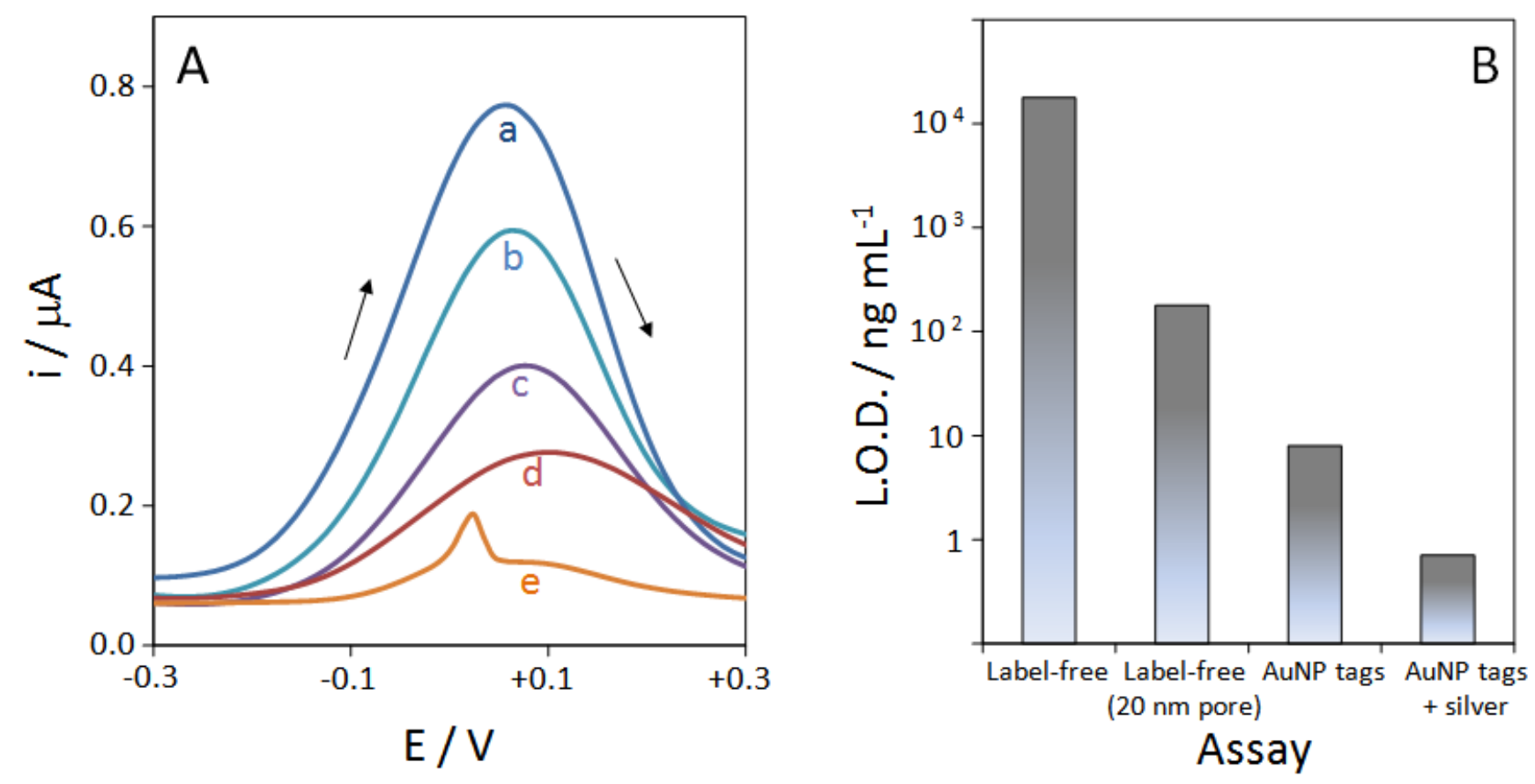


\section{FIGURE 2}
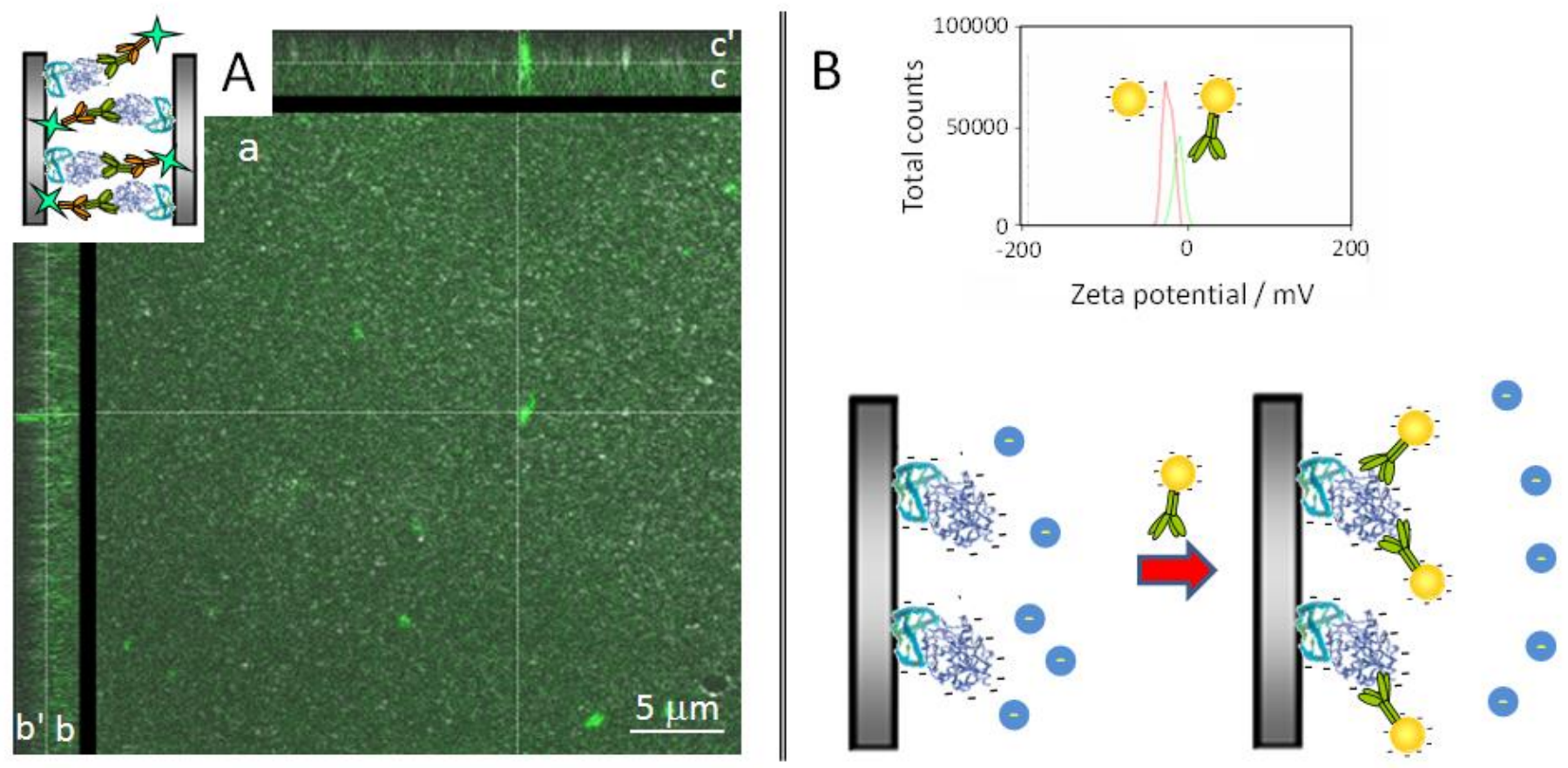


\section{FIGURE 3}
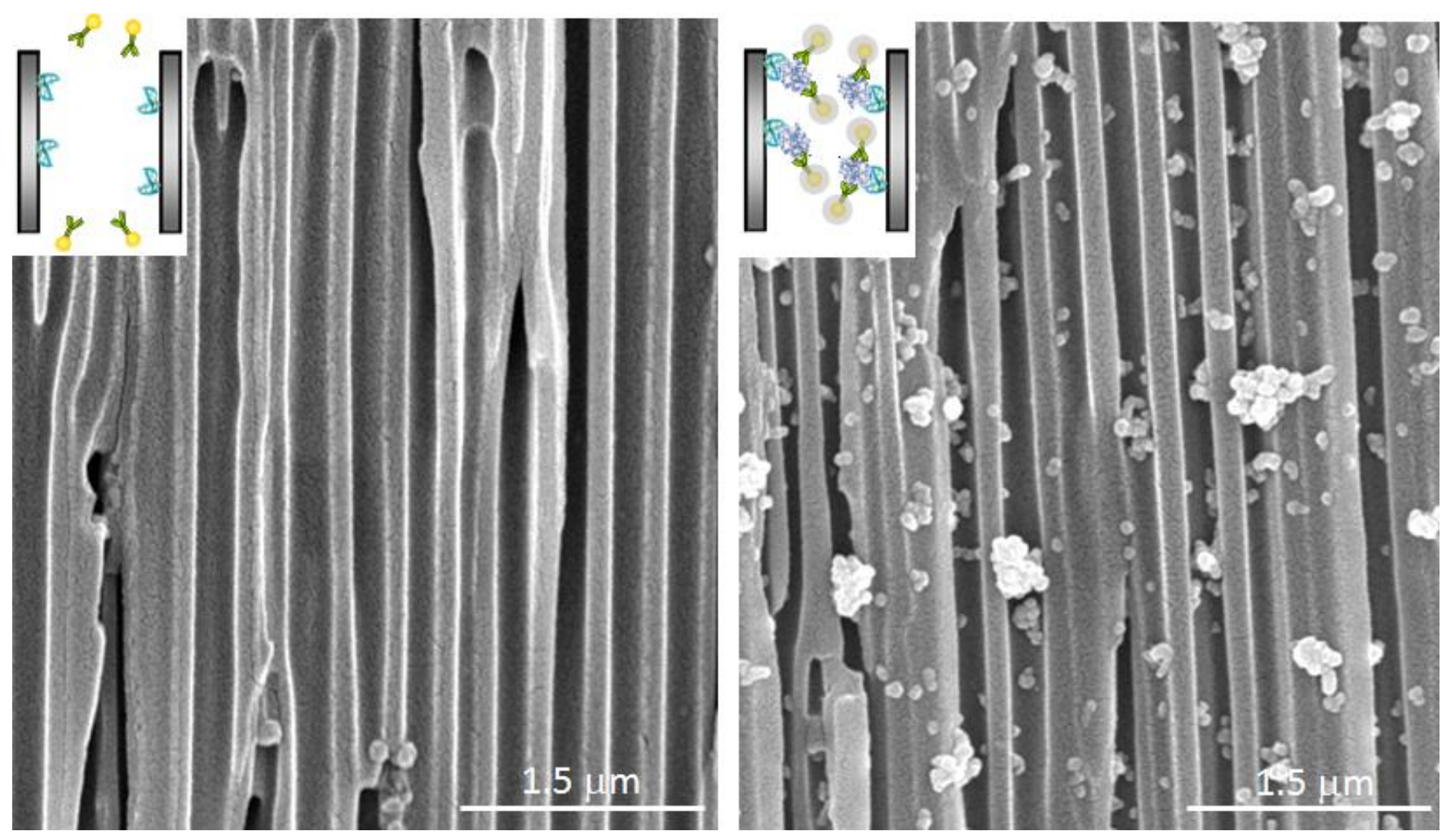


\section{FIGURE 4}
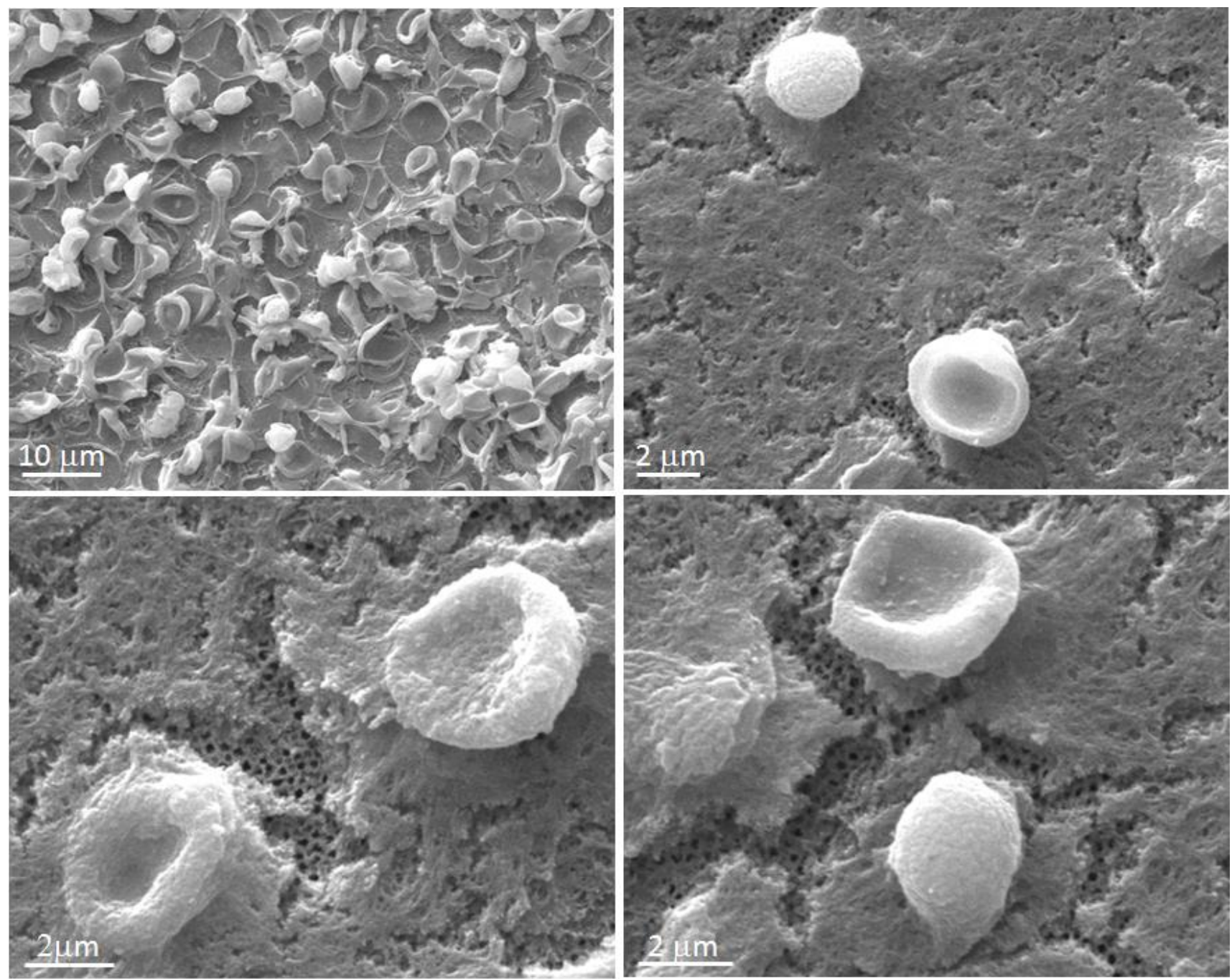


\section{FIGURE 5}
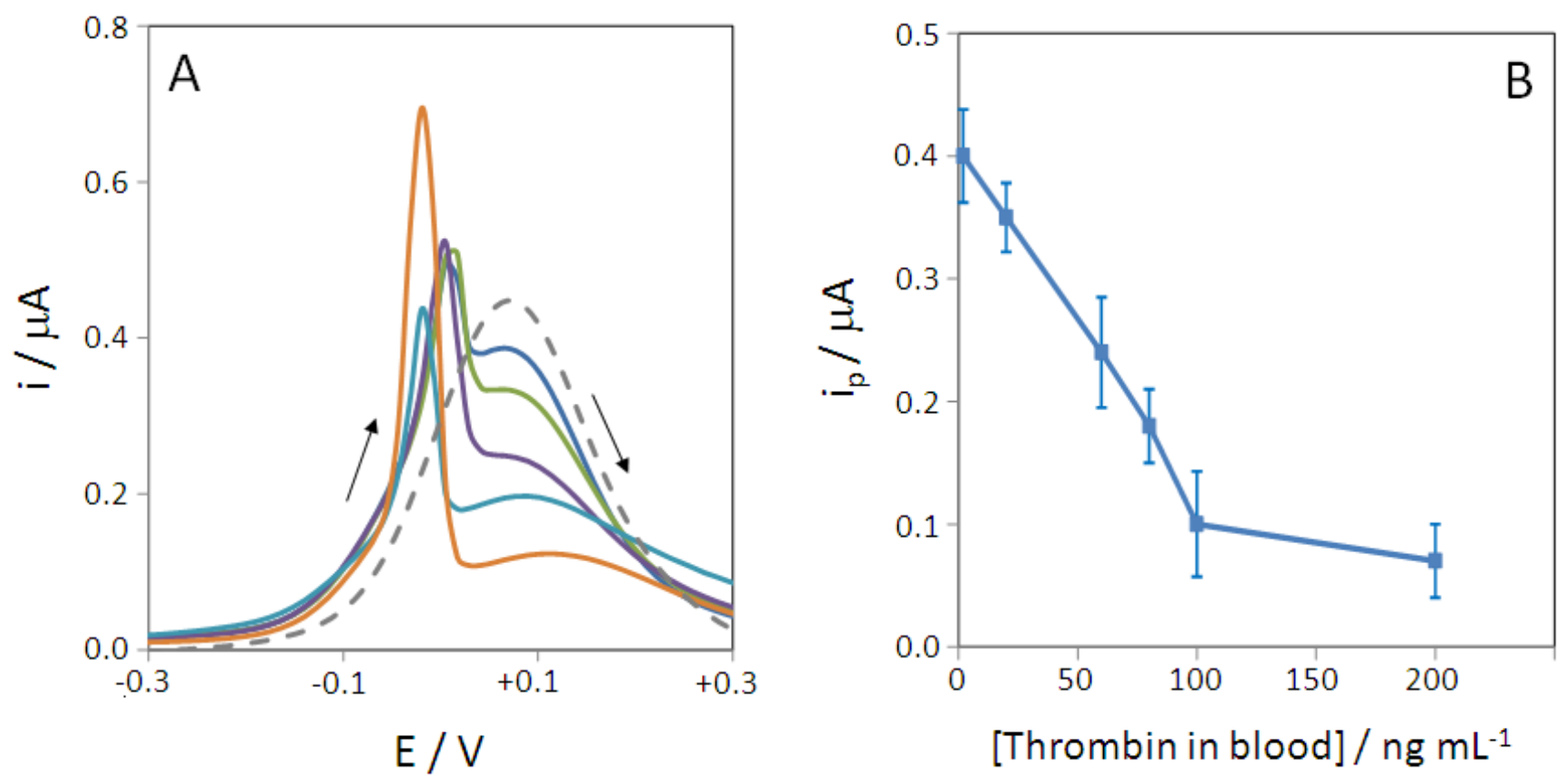


\section{Supplementary material}

\section{Nanochannels for diagnostic of thrombin-related diseases in human blood}

Alfredo de la Escosura-Muñiz ${ }^{1}$, Wilanee Chunglok ${ }^{2}$, Werasak Surareungchai ${ }^{2}$, Arben

$$
\text { Merkoçi }{ }^{1,3^{*}}
$$

${ }^{1}$ Nanobioelectronics \& Biosensors Group, Institut Català de Nanotecnologia, Campus UAB, Bellaterra (Barcelona), Spain.

${ }^{2}$ School of Bioresources \& Technology, King Mongkut's University of Technology, Thonburi, Bangkhuntien Campus, Bangkok, Thailand.

${ }^{3}$ ICREA, Barcelona, Spain

Phone number: +34935868014 ; Fax number: +34935868020

E-mail: arben.merkoci@icn.cat 


\section{Chemicals}

Potassium hexacyanoferrate $\left(\mathrm{K}_{3}\left[\mathrm{Fe}(\mathrm{CN})_{6}\right]\right)$, glutaraldehyde solution $25 \%$ and acetone were purchased from Panreac (Spain). Sodium nitrate $\left(\mathrm{NaNO}_{3}\right)$ was purchased from Sigma (Spain), 3-amino-propiltrimethoxysilane (APS) and propylamine were provided by Fluka (Spain). All reagents were of analytical reagent grade and used without further purification and all the aqueous solutions were prepared in Milli-Q water.

\section{Optical apparatus}

Optical characterizations of the AAO filter membranes were performed using a scanning electron microscope -SEM- (Zeiss EVO® MA 10, Carl Zeiss Microscopy, International Germany) and a Confocal Laser Microscope (TCS SP2 AOBS, Leica, Germany).

\section{Fabrication of screen-printed carbon electrodes (SPCEs)}

The electrochemical transducers used were homemade screen-printed carbon electrodes (SPCEs), consisting of three electrodes: working electrode, reference electrode and counter electrode in a single strip fabricated with a semi-automatic screen-printing machine DEK248 (DEK International, Switzerland). The reagents used for this process were: Autostat HT5 polyester sheet (McDermid Autotype, UK) and Electrodag 423SS carbon ink, Electrodag 6037SS silver/silver chloride ink and Minico 7000 Blue insulating ink (Acheson Industries, The Netherlands). The full size of the sensor strip was $29 \mathrm{~mm} \times 6.7 \mathrm{~mm}$, and the WE diameter was $3 \mathrm{~mm}$. The fabrication of the SPCEs was carried out in three steps. First, a graphite layer was printed onto the polyester sheet, using the screen-printing machine with the stencil (where it is the electron pattern). After curing for 15 minutes at $95^{\circ} \mathrm{C}$, an $\mathrm{Ag} / \mathrm{AgCl}$ layer was printed and cured for 15 minutes at $95^{\circ} \mathrm{C}$. Finally, the insulating ink was printed and cured at $95^{\circ} \mathrm{C}$ for 20 minutes. 
Figure S1 shows images of the 45-sensor sheet obtained following the detailed experimental procedure.

\section{AAO membranes silanization and generation of carbonyl groups}

AAO filter membranes were boiled in Milli-Q water for $1 \mathrm{~h}$, dried in Argon and then immersed into a 5\% APS solution in acetone for $1 \mathrm{~h}$. After thorough washing in acetone and baking at $120^{\circ} \mathrm{C}$ for $30 \mathrm{~min}$, the membranes were left overnight in $25 \%$ aqueous solution of glutaraldehyde, followed by washing in Milli-Q water. See a scheme of the experimental procedure in figure S5.

Finally, after the aptamer immobilization (detailed in the main manuscript text) the glutaraldehyde was neutralized overnight in $10^{-5} \mathrm{M}$ aqueous solution of propylamin, in order to improve the further aptamer recognition efficiency.

\section{Conjugation of AuNPs to anti-thrombin IgG antibodies}

The conjugation of AuNPs to anti-thrombin antibodies was performed according to the following procedure [; De la Escosura-Muñiz et al. Biosen. Bioelectron. 24 (2009) 24752482]. The amount of antibodies necessary to obtain the total coverage of the AuNPs was optimized through the gold aggregation test [A. Ambrosi et al. Anal. Chem. 79 (2007) 5232-5240].

AuNP suspension $(2 \mathrm{~mL})$ was mixed with the antibody solution $\left(100 \mu \mathrm{L}, 100 \mu \mathrm{g} \mathrm{mL}^{-1}\right)$ and incubated at $25^{\circ} \mathrm{C}$ for $20 \mathrm{~min}$. Subsequently, a blocking step with bovine serum albumin (BSA; $150 \mu \mathrm{L}, 1 \mathrm{mg} \mathrm{mL}^{-1}$ ) by incubating at $25^{\circ} \mathrm{C}$ for $20 \mathrm{~min}$ was undertaken. Finally, centrifugation at $4^{\circ} \mathrm{C}$ and $14000 \mathrm{rpm}$ for $20 \mathrm{~min}$ was carried out, and anti-thrombin IgG/AuNPs conjugates were reconstituted in PBS solution. 


\section{Optimization of the aptamer/thrombin interaction time}

The aptamer/thrombin interaction time was optimized for the $200 \mathrm{~nm}$ pore sized membranes in the label-free assay, fixing a concentration of thrombin of $100 \mu \mathrm{gL}^{-1}$ and keeping the rest of the experimental conditions detailed in the materials and methods section of the main text. The values of the voltammetric peak current at $+0.10 \mathrm{~V}$ (analytical signal) registered for incubation times of 10, 20, 30, 45, 60 and 90 minutes are shown in figure S2. As it can be observed, when higher is the incubation time higher is the analytical signal reaching a plateau at 60 minutes. This time was consequently chosen as optimum for the experimental procedure.

\section{Sample preparation for SEM analysis}

$30 \mu \mathrm{L}$ of the blood sample were placed on the filtering side of the AAO filter membranes and left in a petri dish for 60 minutes. After that, the membranes were immersed in $2.5 \%$ glutaraldehyde in order to fix the cells there overnight. After that, the samples were dehydrated sequentially in ethanol of different purities $\left(1 \mathrm{X} 50^{\circ}, 2 \mathrm{X} 70^{\circ}, 3 \mathrm{X} 90^{\circ}, 3 \mathrm{X} 95^{\circ}\right.$, all these steps consisted in immersion during 10 minutes; $3 \mathrm{X} 100^{\circ}$, these steps were perfomed during 20 minutes). After that, the membranes were immersed in etanol/HMDS for 20 minutes and finally in HMDS $100^{\circ}$ (2X, 30 minutes). The excess of HMDS was removed and then the membranes were coated by an sputtering of gold and then analyzed in the SEM. 


\section{Electrochemical cell set-up}

AAO filter membranes were fixed onto the screen-printed carbon electrodes (SPCEs) by a physical attachment inside a methacrylate cell, achieved by placing the SPCE onto a methacrylate block and putting the membrane with the filtering side up covering in this way the three electrodes surface. Then, a second methacrylate block containing a hole of the same size as the working area of the SPCE was placed onto the membrane, using an insulating O-ring between them to avoid liquid leakage and finally the system is fixed using screws. In this way, a $200 \mu \mathrm{L}$ electrolytic cell is defined (see figure S3). 


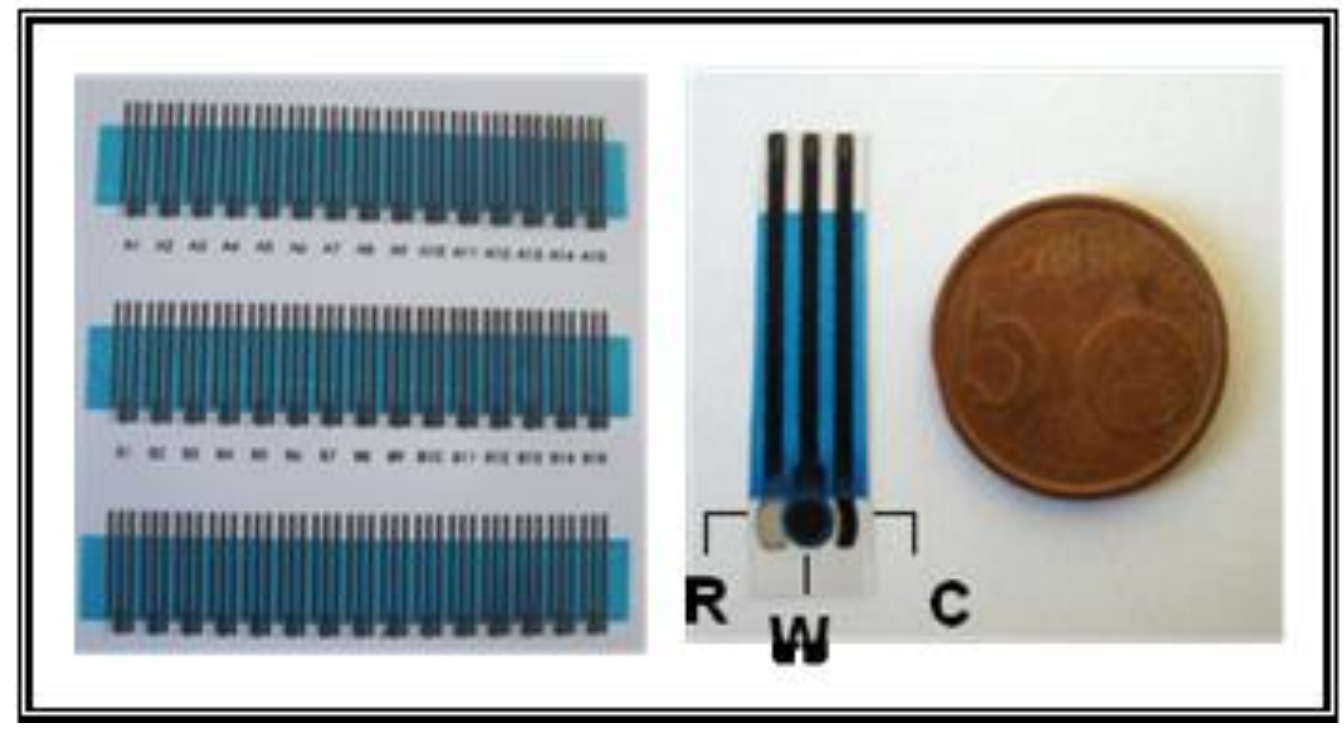

Figure S1. (Left) Images of the 45 SPCE sensors sheet obtained following the detailed experimental procedure. (Right) Detail of one SPCE, containing the three electrodes in the working area: $\mathrm{R}-\mathrm{Ag} / \mathrm{AgCl}$ reference electrode, $\mathrm{W}$ - carbon working electrode and $\mathrm{C}$ - carbon counter electrode. 


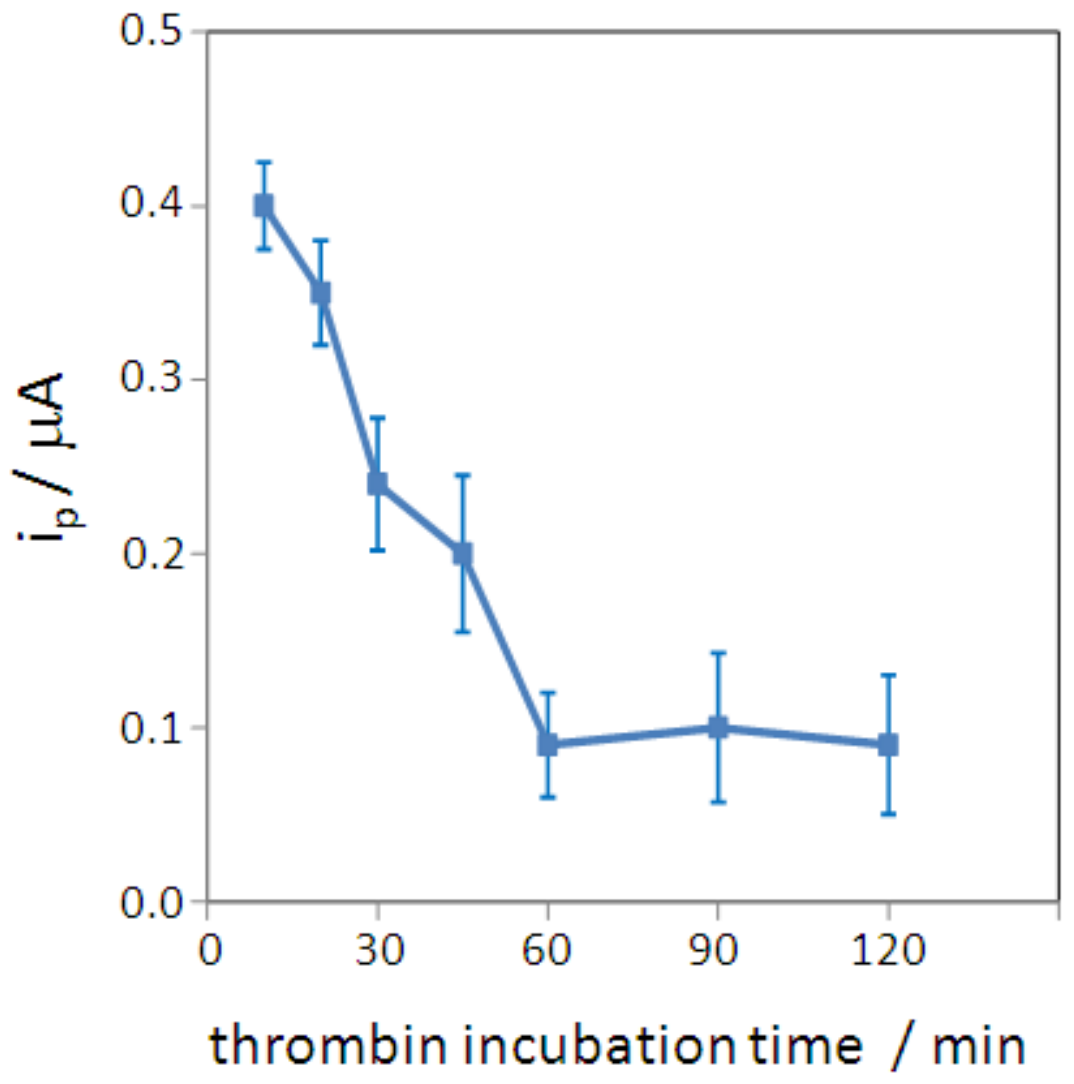

Figure S2. Effect of the aptamer/thrombin incubation time on the voltammetric peak current chosen as analytical signal. Thrombin concentration: $100 \mu \mathrm{g} \mathrm{mL}^{-1}$. Label-free assay. Membranes pore size: $200 \mathrm{~nm}$. The rest of the experimental conditions are the detailed in the materials and methods section of the main text. 


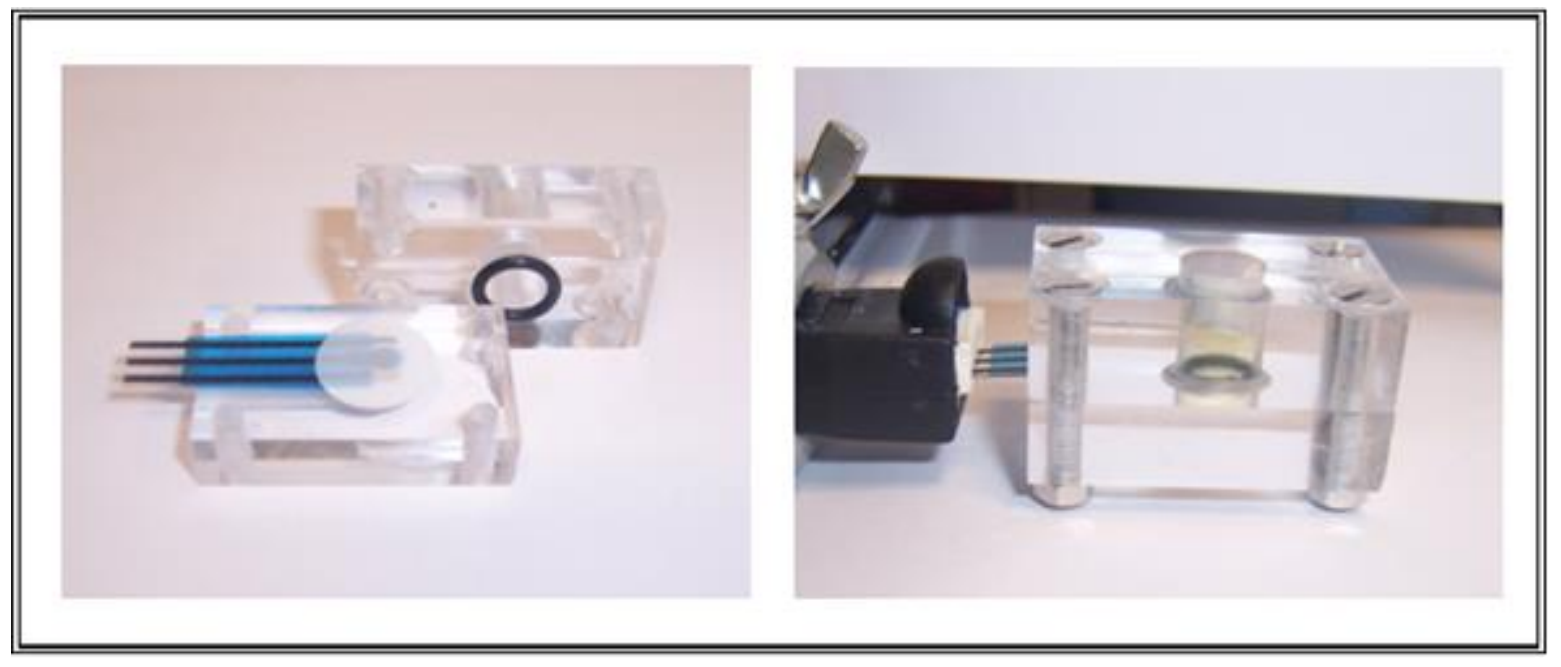

Figure S3. (Left) Picture of the SPCE placed into the two methacrylate blocks with the AAO filter membrane covering the working area. (Right) Cell assembled, filled with $1 \mathrm{mM}$ $\mathrm{K}_{3}\left[\mathrm{Fe}(\mathrm{CN})_{6}\right] / 0.1 \mathrm{M} \mathrm{NaNO}_{3}$ solution and connected to the potentiostat. 

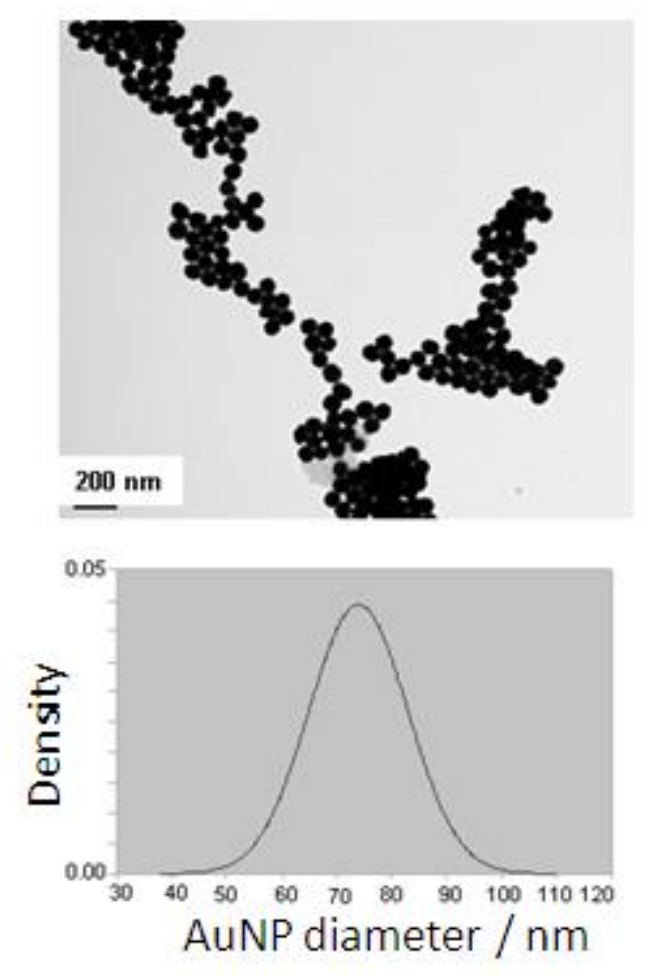

Figure S4. TEM image obtained for the AuNPs used as tags (above) and the corresponding size distribution (below). 

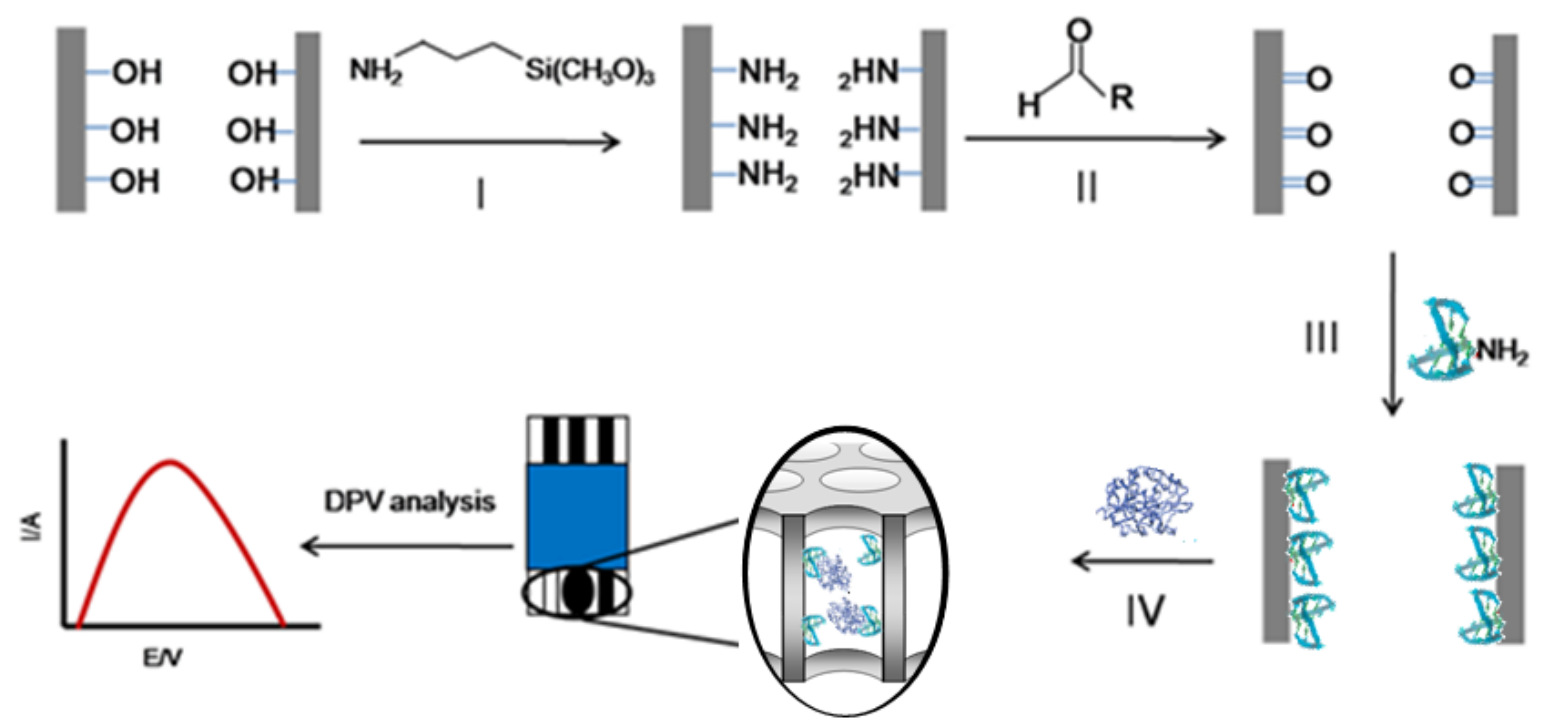

Figure S5. . Schematic representation of the processes involved for the nanochannels functionalization, immobilization of the aptamer, reaction with thrombin and electrochemical measurement: (I) generation of amino groups on internal walls by silanization reaction with 3-amino propiltrimethoxysilane (APS); (II) generation of carboxyl groups by reaction with glutaraldehyde; (III) immobilization of aptamer by petide bond; (IV) reaction with aptamer. 

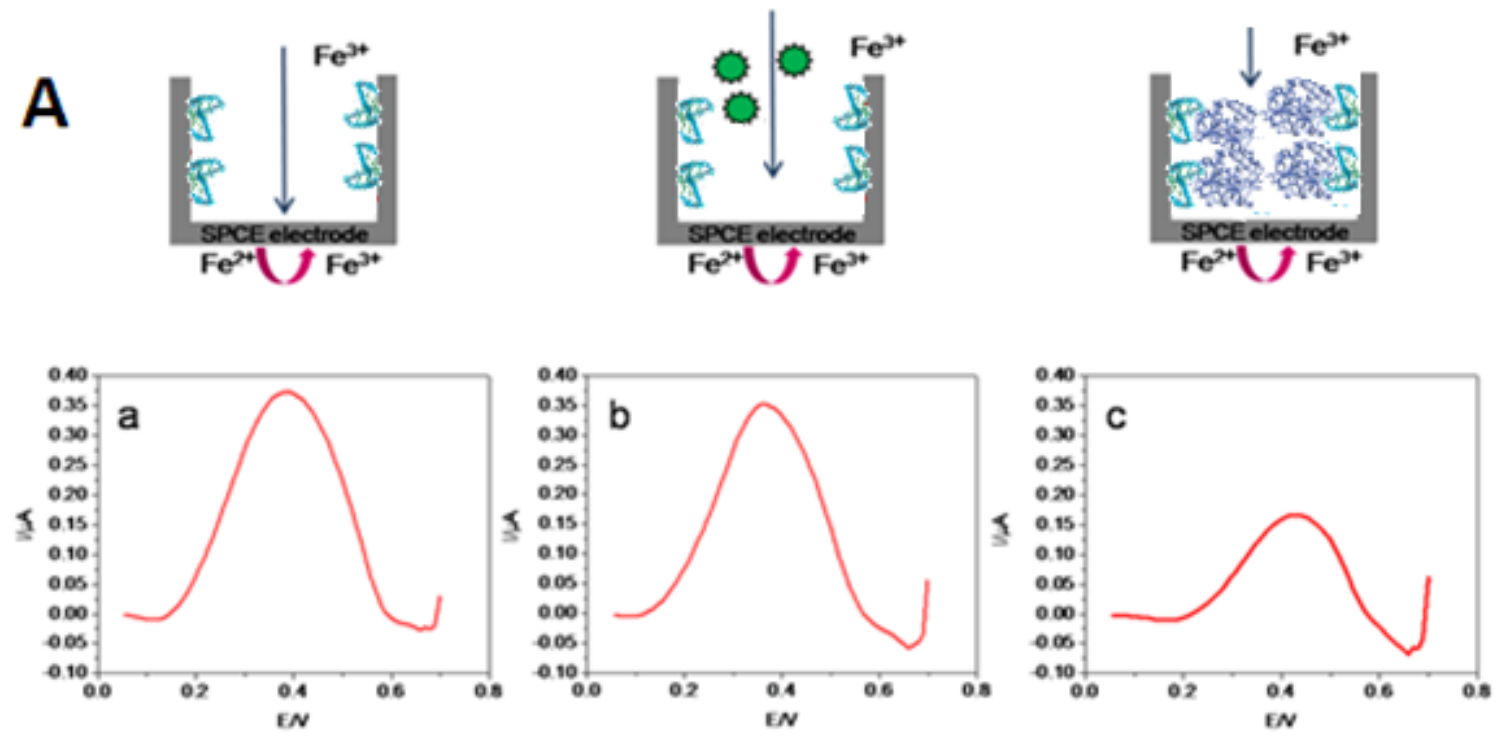

B

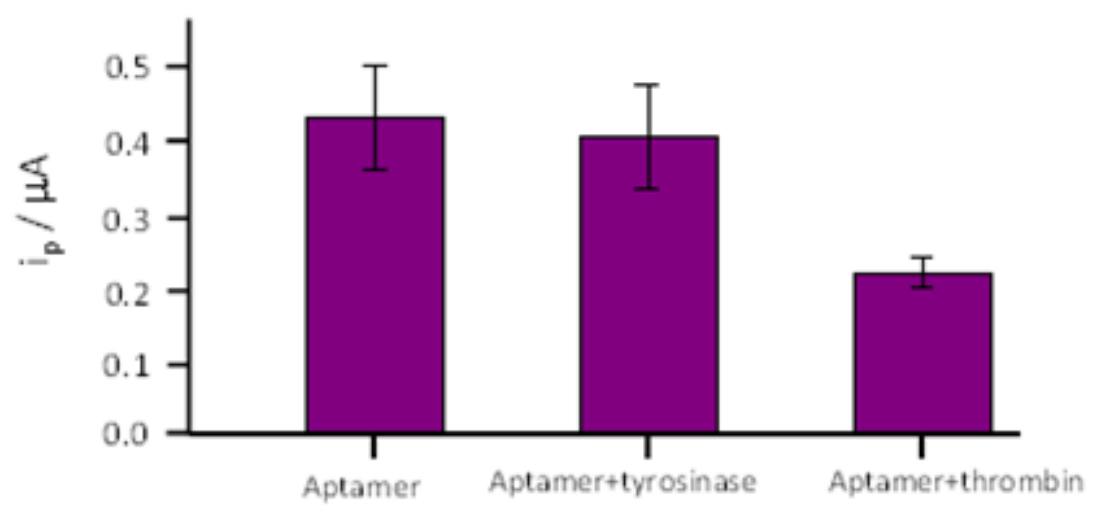

Figure S6. (A) Scheme of the process which occurs inside the nanochannels and the corresponding DPVs obtained in $1 \mathrm{mM} \mathrm{K} \mathrm{K}_{3}\left[\mathrm{Fe}(\mathrm{CN})_{6}\right] / 0.1 \mathrm{M} \mathrm{NaNO}_{3}$ for AAO filter membranes with $200 \mathrm{~nm}$ pore sizes after the aptamer immobilization (a) the reaction with 1 $\mathrm{mg} \mathrm{ml}^{-1}$ of tyrosinase (b) and the reaction with the same concentration of thrombin (c). (B) Summary of the obtained results. 